\title{
Taxonomic significance of stomatal complex in fifteen species of Dendrobium Swartz (Orchidaceae) of Nepal
}

\author{
Baba Maiya Pradhan ${ }^{1} \bowtie$ and Devendra M. Bajracharya ${ }^{2}$ \\ 1Department of Botany, Patan Multiple Campus, Tribhuvan University, Nepal \\ ${ }^{2}$ Amrit Science Campus, Tribhuvan University, Nepal \\ baba.m.pradhan@gmail.com
}

\begin{abstract}
Dendrobium Swartz is one of the largest and most problematic genus of the tribe Dendrobieae of the family Orchidaceae. Nepal harbors about thirty-one species of Dendrobium. Micromorphological characters like the stomatal complex in fifteen species of Dendrobium Swartz (Orchidaceae) of Nepal were studied for understanding the taxonomic significance of stomatal complex within the genus. For the study of stomatal complex of leaf, Carpenter's method was followed. Stomatal index and stomatal frequency were calculated using formula given by Salisbury. The terminologies used for the stomatal complex type were followed those of Patel. In all species the leaf surface was found hypostamic. Tetracytic (a-tetra-monocyclic, b-tetra-monocyclic and c-tetra-monocyclic) and eupara twi-monocyclic with hexa-monocyclic types of stomata were recorded in the genus. Tetracytic type was found in fourteen species and eupara twi-monocyclic with hexa-monocyclic was found in only one species. The elliptical shape of stomata was found in more species than the circular shape. The epidermal cells of leaf were found rectangular, square, polygonal to irregular. The distribution of stomata on leaf surface, type of stomata and shape of epidermal cells were found to be taxonomically significant. These characters could be used as distinguishing characters to delimit the species in the genus.
\end{abstract}

Keywords: anatomical key, Dendrobium, epidermal cells, micromorphological character, stomatal variation

\section{INTRODUCTION}

The family Orchidaceae is widely distributed in the tropical and subtropical regions of the world and is regarded as one of the largest, most diverse and distinctive families of flowering plants (Burns-Balogh \& Funk, 1986; Dressler, 1981, 1993). Dendrobium Swartz is a dominant genus of the tribe Dendrobieae of the family Orchidaceae. The genus was established by Olof Swartz (1799) based on Dendrobium moniliforme (L.) Sw. as the type species of the genus. The genus Dendrobium Swartz consists of about 900 species, widely distributed in China, Japan, India, Malaysia, Indonesia to New Guinea, Australia, New Zealand and the Pacific islands (Seidenfaden, 1985; Pearce \& Cribb, 2002). In Nepal they are distributed from tropical to alpine regions. Hara et al. (1978) and Press et al. (2000) recorded 26 species of Dendrobium from Nepal. However, Rokaya et al. (2013) enumerated 31 species of Dendrobium from Nepal Himalaya. Species of Dendrobium are mostly epiphytes with some 
are lithophytes. The distinguishing characters of the genus Dendrobium are presence of cane-like stem with pseudobulb covered with or without sheathing leaf bases, distichous or duplicate leaves, spiral flowers with basally jointed lips with prominent spurs formed by the column foot and naked pollinia.

Micromorphological study on leaf epidermal surface had been widely used in taxonomic and phylogenetic studies as they can provide valuable information (Patel, 1979; Stace, 1980; Davis, 1997). The notable work on stomatal patterns in some angiosperms had studied by Stebbins \& Khush (1961), and Croxdale (2000). Leaf epidermal anatomy particularly of stomata was reported to provide valuable taxonomic and systematic evidence in both living and fossil plants (Wilkinson, 1979; Kong, 2001). In Orchidaceae, several micromorphological studies on stomatal types based on their structures have been done by Kaushik (1983) and Khasim \& Rao (1990). Singh \& Singh (1974) studied the organization of stomatal complex in family Orchidaceae. Kaushik (1983) and Rasmussen (1987) studied the ecological significance of dermal characters. Yukawa et al. (1992) reported the existence of two types of stomatal shape in the genus Dendrobium and its systematic significance. Arditti (1992) suggested that the shape of subsidiary cells had been used to distinguish the stomatal complex of Orchidaceae into several types. Vij et al. (1991) also examined epidermal feature of Indian orchids for taxonomic and ecological implications. Where Himalayan species are concerned, among the twelve species of the genus Oberonia, eight types of stomata had classified according to the arrangement of subsidiary cells (Shakya, 1999). In case of Himalayan genus Eria seven types of stomata complex has been studied (Bajracharya, 2005). The stomatal types had been reported to be significant in establishing the various ranks and clarifying the interrelationship among the different taxa and also resolving many taxonomic problems (Baranova, 1992).

Dendrobium is one of the largest and most problematic genera with respect to its intrageneric classification and relationships to other taxa in the family orchidaceae. The large number of species and diversity of gross morphology are the most prominent reasons for the complexity of Dendrobium taxonomy and the confusion over its intrageneric relationships. Under such circumstances orchid systematics needs a thorough revision using parameters other than gross morphology to clarify the intrageneric relationships among Dendrobium species. So in the present study the preliminary attempts were made on the study of distribution of stomata on leaf surface, stomatal types and epidermal cell types to clarify the intrageneric relationship among these taxa.

\section{MATERIALS AND METHODS}

In present study 15 species of Dendrobium Swartz viz. D. amoenum Wall. ex Lindl., D. anceps Sw., D. aphyllum (Roxb.) C.E.C. Fisch., D. bicameratum Lindl., D. chrysanthum Wall. ex Lindl., D. crepidatum Lindl. \& Paxton, D. densiflorum Lindl., D. denudans D. Don., D. erifforum Griff., $D$. formosum Roxb. ex Lindl., $D$. heterocarpum Wall. ex Lindl., D. longicornu Lindl., $D$. moniliforme (L.) Sw., D. moschatum (Buch.-Ham.) Sw., D. transparens Wall.ex Lindl. were studied. The study was based on the live specimens. Field visits were carried out in different parts of Nepal to collect the live specimens. Live specimens were identified by studying protologues, type specimens and authentic herbarium specimens deposited at $\mathrm{KATH}, \mathrm{TUCH}$, $\mathrm{CAL}, \mathrm{BM}, \mathrm{K}, \mathrm{P}$ and TI. Protologues and images of type specimens have been downloaded 
from different websites including www.botanicus.org, www.biodiversitylibrary.org, www.plants. jstor.org, www.kew.org and www.nybg.org. Update nomenclature of Dendrobium species have been acquired from websites www.kew.org.wcsp/monocots and www.theplantlist.org.

The study of stomata was done on the fresh leaves of Dendrobium species collected from various localities of Nepal Himalaya. For the study of stomata, the method described by Carpenter (2005) with some modification was followed. Stomatal index and stomatal frequencies were calculated using the formula given by Salisbury (1928). The terminologies used for stomatal complex types were followed those of Patel (1979).

\section{RESULTS AND DISCUSSION}

The stomatal complex of 15 species of Dendrobium had examined with light microscope. The species of Dendrobium were found hypostamic leaf surfaces (i.e., stomata occurring on the abaxial surface only). The stomata were largely of tetracytic type (a, b and c-tetra-monocyclic) whereas eupara twi-monocyclic with hexa-monocyclic rarely present in some species. The stomata possess four to five subsidiary cells.

The stomatal distribution on the leaf surface, stomatal size with guard cells, stomatal pore size, stomatal frequency, stomatal index, shape of stomata, type of stomata, shape and type of epidermal cells on the abaxial surface of leaves was found to be variable in different species of Dendrobium (table 1).

The epidermal cells on abaxial surface are rectangular to polygonal and regular to irregular in shape. In most of species like $D$. amoenum, $D$. anceps, $D$. aphyllum, $D$. eriiflorum, $D$. formosum, $D$. heterocarpum, $D$. longicornu, $D$. moniliforme, $D$. moschatum and $D$. transparens the epidermal cells were found to be rectangular to polygonal where as in species like $D$. bicameratum, $D$. chrysanthum, $D$. crepidatum, $D$. densiflorum and $D$. denudans the epidermal cells were found to be squarish, pentagonal, hexagonal to polygonal and irregular size in some species with anticlinal wall varying from straight-sided to curvilinear. The epidermal cells were mostly parenchymatous (thin-walled or thick-walled) in species like $D$. amoenum, $D$. anceps, $D$. aphyllum, D. bicameratum, $D$. chrysanthum, 
Pradhan and Bajracharya: Taxonomic significance of stomatal

\begin{tabular}{|c|c|c|c|c|c|c|c|c|c|c|c|c|c|c|c|c|}
\hline & 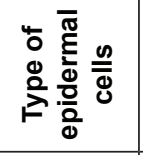 & 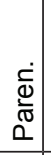 & $\begin{array}{l}\frac{c}{d} \\
\frac{d}{\sigma} \\
\square\end{array}$ & $\begin{array}{l}\bar{\Phi} \\
\frac{\Phi}{\widetilde{\sigma}} \\
\square\end{array}$ & $\begin{array}{l}\bar{\Phi} \\
\frac{\Phi}{\sigma} \\
0 \\
\end{array}$ & $\begin{array}{l}\frac{c}{d} \\
\frac{d}{\sigma} \\
\square\end{array}$ & 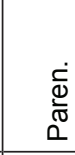 & 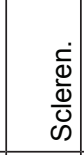 & 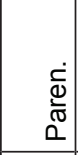 & 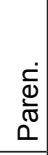 & $\begin{array}{l}\frac{\mathrm{c}}{\mathbf{d}} \\
\frac{\mathrm{d}}{\mathrm{d}} \\
\mathrm{S}\end{array}$ & 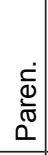 & $\begin{array}{l}\frac{\sigma}{\Phi} \\
\stackrel{\varpi}{\sigma} \\
\square\end{array}$ & $\begin{array}{l}\bar{\complement} \\
\frac{\bar{d}}{\mathbb{d}} \\
\square\end{array}$ & $\begin{array}{c}\bar{\Phi} \\
\frac{\Phi}{\sigma} \\
0\end{array}$ & 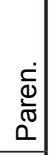 \\
\hline 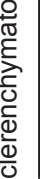 & 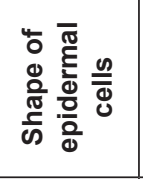 & 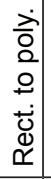 & 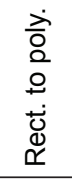 & 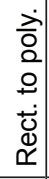 & $\begin{array}{l}+ \\
\stackrel{0}{0} \\
\stackrel{\infty}{0} \\
\infty \\
\infty\end{array}$ & $\begin{array}{l}\frac{\pi}{2} \\
\dot{\bar{c}} \\
\dot{c}\end{array}$ & 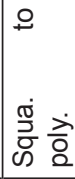 & 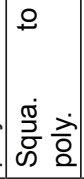 & 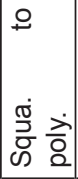 & 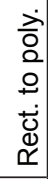 & 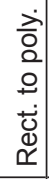 & 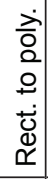 & 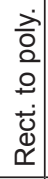 & 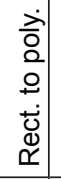 & 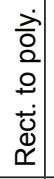 & $\begin{array}{l}\dot{\vec{a}} \\
\dot{0} \\
\stackrel{0}{0} \\
\dot{0} \\
\dot{0} \\
\dot{0}\end{array}$ \\
\hline en & 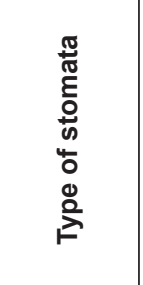 & $\begin{array}{l}\frac{0}{0} \\
0 \\
0 \\
0 \\
0 \\
\frac{1}{1} \\
\frac{0}{0} \\
\frac{0}{0} \\
\frac{1}{0}\end{array}$ & 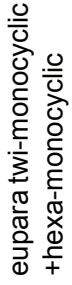 & 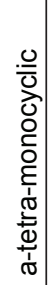 & 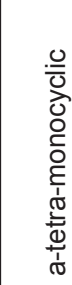 & 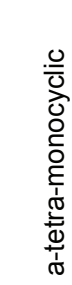 & 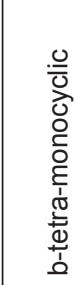 & $\begin{array}{l}\frac{0}{0} \\
\overline{0} \\
0 \\
0 \\
0 \\
\frac{0}{1} \\
\frac{0}{0} \\
\frac{0}{0} \\
\frac{1}{0}\end{array}$ & $\begin{array}{l}\frac{0}{0} \\
\overline{0} \\
0 \\
0 \\
0 \\
\frac{0}{1} \\
\frac{0}{0} \\
\frac{0}{0} \\
\frac{1}{0}\end{array}$ & 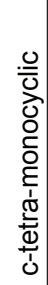 & 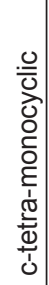 & 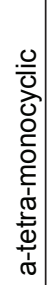 & 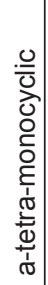 & $\begin{array}{l}\frac{0}{0} \\
0 \\
0 \\
0 \\
0 \\
\frac{\varepsilon}{1} \\
\frac{1}{0} \\
\frac{0}{0} \\
\frac{1}{0}\end{array}$ & 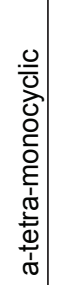 & 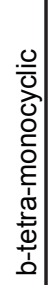 \\
\hline $\begin{array}{l}\text { II } \\
\frac{5}{11} \\
\text { II }\end{array}$ & 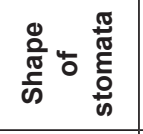 & $\begin{array}{l}\stackrel{0}{\bar{z}} \\
\stackrel{\overline{\bar{u}}}{\bar{w}}\end{array}$ & $\frac{.0}{\stackrel{\underline{\underline{n}}}{\overline{\bar{w}}}}$ & 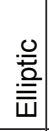 & 을 & 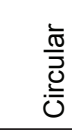 & 을 & 毫 & $\frac{.0}{\stackrel{0}{\bar{z}}}$ & 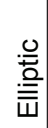 & $\begin{array}{l}\frac{\pi}{\bar{T}} \\
\frac{\mathrm{U}}{\bar{U}} \\
\end{array}$ & $\begin{array}{l}\frac{\bar{\omega}}{\bar{J}} \\
\frac{\bar{U}}{\bar{U}}\end{array}$ & $\frac{.0}{\overline{\underline{n}}}$ & $\begin{array}{l}\frac{1}{\bar{T}} \\
\frac{0}{U} \\
\dot{U}\end{array}$ & $\begin{array}{l}\frac{\bar{d}}{\bar{J}} \\
\frac{\bar{J}}{\bar{U}} \\
\end{array}$ & 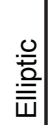 \\
\hline م. & 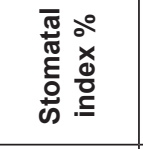 & $\begin{array}{l}\bar{\infty} \\
0 \\
1\end{array}$ & $\begin{array}{l}\stackrel{2}{\rho} \\
m\end{array}$ & $\stackrel{\bar{m}}{\sim}$ & $\stackrel{\text { O }}{\Lambda}$ & $\begin{array}{l}\infty \\
0 \\
i \\
i \\
\end{array}$ & $\begin{array}{l}\text { ठ } \\
\stackrel{m}{\rightleftharpoons}\end{array}$ & \begin{tabular}{l}
0 \\
\multirow{0}{0}{} \\
0
\end{tabular} & $\begin{array}{l}\bar{T} \\
\stackrel{0}{\circ} \\
\leftarrow\end{array}$ & $\begin{array}{l}8 \\
0 \\
0 \\
\end{array}$ & $\begin{array}{l}0 \\
\stackrel{\sim}{\sim} \\
\end{array}$ & $\begin{array}{l}\dot{U} \\
\dot{m} \\
\stackrel{5}{-}\end{array}$ & $\begin{array}{l}03 \\
0 \\
0 \\
\circ \\
\end{array}$ & $\stackrel{\stackrel{0}{+}}{\stackrel{\sim}{\leftarrow}}$ & 이 & $\begin{array}{l}\bar{\kappa} \\
\stackrel{0}{0} \\
\end{array}$ \\
\hline$\frac{\infty}{0}$ & 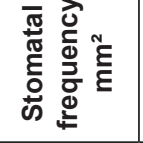 & 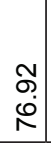 & $\stackrel{6}{\stackrel{0}{\phi}}$ & $\begin{array}{l}L \\
\dot{6} \\
\dot{g}\end{array}$ & $\begin{array}{l}\text { న్ } \\
\text { ê } \\
\end{array}$ & 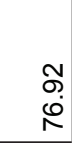 & $\begin{array}{l}\text { N } \\
\text { ò } \\
\stackrel{9}{\leftarrow}\end{array}$ & $\begin{array}{l}\tilde{N} \\
\dot{\rho} \\
\end{array}$ & ๙ூ. & $\begin{array}{l}\stackrel{0}{0} \\
\stackrel{N}{\sim}\end{array}$ & $\begin{array}{l}N \\
\sigma \\
e \\
\end{array}$ & 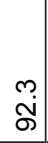 & 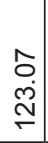 & $\begin{array}{c}n \\
\infty \\
\infty \\
\stackrel{m}{\sigma}\end{array}$ & $\begin{array}{l}N \\
\sigma \\
e \\
\hat{\nu}\end{array}$ & 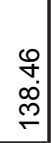 \\
\hline 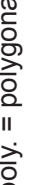 & 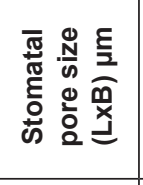 & 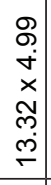 & 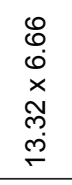 & 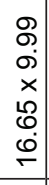 & 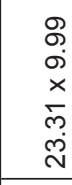 & $\begin{array}{l}0 \\
0 \\
0 \\
\dot{0} \\
\times \\
0 \\
0 \\
0 \\
\dot{0}\end{array}$ & 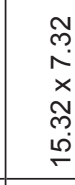 & 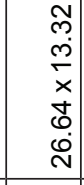 & $\begin{array}{l}\text { o } \\
o \\
\times \\
\times \\
\dot{0} \\
\dot{0} \\
\text { i }\end{array}$ & 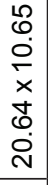 & $\begin{array}{l}0 \\
0 \\
\dot{0} \\
\times \\
\dot{+} \\
\dot{+} \\
\dot{v}\end{array}$ & $\begin{array}{l}0 \\
\dot{0} \\
0 \\
\dot{x} \\
0 \\
0 \\
\dot{0} \\
\end{array}$ & 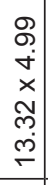 & $\begin{array}{l}0 \\
\dot{0} \\
\dot{0} \\
\times \\
\dot{0} \\
\dot{0} \\
\dot{0} \\
\end{array}$ & $\begin{array}{l}8 \\
8 \\
0 \\
\times \\
\times \\
0 \\
\dot{0} \\
0\end{array}$ & 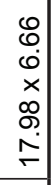 \\
\hline 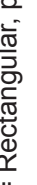 & 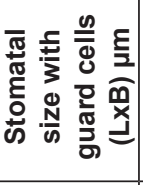 & 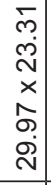 & 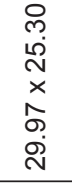 & 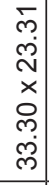 & 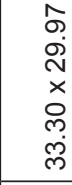 & 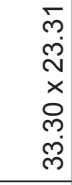 & $\begin{array}{l}\text { பे } \\
\dot{\leftrightarrow} \\
\text { N } \\
\times \\
\end{array}$ & 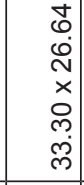 & 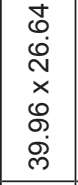 & 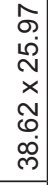 & 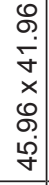 & 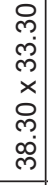 & $\begin{array}{l}\bar{n} \\
\tilde{N} \\
\times \\
\times \\
\hat{\sigma} \\
\dot{D} \\
\end{array}$ & $\begin{array}{l}8 \\
\dot{0} \\
\dot{j} \\
\times \\
\dot{\rho} \\
m \\
\dot{m} \\
\ddot{m}\end{array}$ & 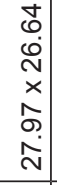 & 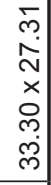 \\
\hline U. & 恋 & $\begin{array}{l}\overline{.} \\
\cdot \frac{\pi}{x} \\
\mathbb{0} \\
\frac{0}{\alpha} \\
\end{array}$ & $\begin{array}{l}\bar{\pi} \\
\frac{\pi}{x} \\
\mathbb{0} \\
\frac{\pi}{4}\end{array}$ & $\begin{array}{l}\overline{-\pi} \\
\bar{x} \\
\bar{\pi} \\
\frac{0}{\alpha} \\
\end{array}$ & 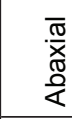 & $\begin{array}{l}\bar{\pi} \\
\frac{\pi}{x} \\
\bar{\pi} \\
\frac{\pi}{\alpha}\end{array}$ & 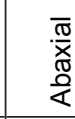 & 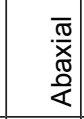 & $\begin{array}{l}\bar{\pi} \\
\frac{\pi}{x} \\
\mathbb{\pi} \\
\frac{\pi}{4} \\
\end{array}$ & $\begin{array}{l}\bar{\pi} \\
\frac{\pi}{x} \\
\frac{\pi}{2} \\
\frac{0}{4} \\
\end{array}$ & $\begin{array}{l}\overline{. \pi} \\
-\frac{\pi}{x} \\
\frac{\pi}{0} \\
\frac{0}{4} \\
\end{array}$ & $\begin{array}{l}\overline{. \pi} \\
\frac{\pi}{x} \\
\mathbb{0} \\
\frac{0}{4} \\
\end{array}$ & 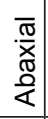 & $\begin{array}{l}\overline{. \pi} \\
\frac{\pi}{x} \\
\mathbb{\widetilde { d }} \\
\frac{0}{4} \\
\end{array}$ & $\begin{array}{l}\bar{\pi} \\
\frac{\pi}{x} \\
\frac{\pi}{0} \\
\frac{\pi}{4}\end{array}$ & $\begin{array}{l}\bar{\pi} \\
\cdot \frac{\pi}{x} \\
\frac{\pi}{\alpha} \\
\frac{0}{4} \\
\end{array}$ \\
\hline$\frac{1}{1}$ & 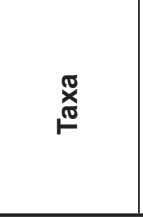 & 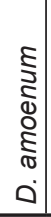 & 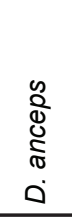 & 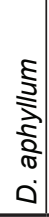 & 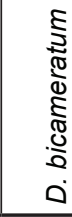 & 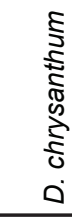 & $\begin{array}{l}\frac{3}{\pi} \\
\frac{0}{2} \\
\frac{0}{0} \\
0 \\
0\end{array}$ & $\begin{array}{l}5 \\
\frac{1}{2} \\
\frac{2}{5} \\
\frac{1}{d} \\
\frac{0}{0} \\
0 \\
0\end{array}$ & 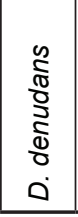 & 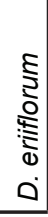 & $\begin{array}{l}5 \\
5 \\
0 \\
\vdots \\
\vdots \\
\vdots \\
\vdots \\
0\end{array}$ & $\begin{array}{l}5 \\
5 \\
\frac{5}{0} \\
0 \\
0 \\
0 \\
0 \\
0 \\
0 \\
0 \\
0\end{array}$ & $\begin{array}{c}\gtrsim \\
\vdots \\
\vdots \\
0 \\
\vdots \\
\vdots \\
0 \\
0\end{array}$ & 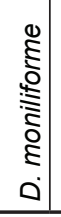 & 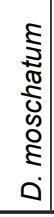 & $\begin{array}{c}0 \\
2 \\
0 \\
0 \\
0 \\
0 \\
0 \\
0 \\
0 \\
0 \\
0 \\
0\end{array}$ \\
\hline
\end{tabular}


The stomata were found mostly tetracytic type ( $\mathrm{a}, \mathrm{b}$ and c-tetra-monocyclic). In species like D. aphyllum (table 1, plate-I C), D. bicameratum (table 1, plate-I D), D. chrysanthum (table 1, plate-I E), D. densiflorum (table 1, plate-I G), D. denudans (table 1, plate-I H), D. heterocarpum (table 1, plate-II B), D. longicornu (table 1, plate-II C), D. moniliforme (table 1, plate-II D) and $D$. moschatum (table 1, plate-II E) a-tetra-monocyclic type was found whereas b-tetra-monocyclic type was found in $D$. amoenum (table 1 , plate-I A), D. crepidatum (table 1 , plate-I F), D. transparens (table 1, plate-II F) and c-tetra-monocyclic type was found in $D$. eriiflorum (table 1, plate-I I) and D. formosum (table 1, plate-II A). Eupara twi-monocyclic with hexa-monocyclic was found in $D$. anceps (table 1, plate-I B).

Shape of stomata in Dendrobium varied from elliptical to circular. Elliptic type of stomata had found more in number than circular type. In species like $D$. amoenum (table 1, plate-I A), $D$. anceps (table 1, plate-I B), D. aphyllum (table 1, plate-I C), D. bicameratum (table 1, plate-I D), D. crepidatum (table 1, plate-I F), D. densiflorum (table 1, plate-I G), D. denudans (table 1, plate-I H), D. eriiflorum (table 1, plate-I I), D. longicornu (table 1, plate-II C) and $D$. transparens (table 1, plate-II F) the stomata were elliptical type whereas the species like $D$. chrysanthum (table 1, plate-I E), D. formosum (table 1, plate-II A), D. heterocarpum (table 1, plate-II B), $D$. moniliforme (table 1, plate-II D) and D. moschatum (table 1, plate-II E) had circular type.

The largest stoma was found in $D$. formosum with the area of $1928.48 \mu \mathrm{m}^{2}$ and the smallest in $D$. amoenum and $D$. longicornu with the area of $698.60 \mu \mathrm{m}^{2}$. The stomatal pore size was found smallest in $D$. amoenum and $D$. longicornu ranging from $66.46 \mu \mathrm{m}$ to largest size $354.84 \mu \mathrm{m}$ in $D$. densiflorum. The stomatal frequency varied from $46.15 \mathrm{~mm}^{2}-138.50 \mathrm{~mm}^{2}$. The highest stomatal frequency was found in $D$. moniliforme with $138.50 \mathrm{~mm}^{2}$ and the lowest in $D$. anceps and $D$. aphyllum with $46.15 \mathrm{~mm}^{2}$. The stomatal index was found highest in $D$. heterocarpum with 13.04 and the lowest in $D$. anceps with 3.75 .

On the basis of micromorphological characters like distribution of stomata on leaf surface, shape of epidermal cell, type of stomata, Dendrobium species of Nepal has been divided into different groups to delimit the taxa.

\section{Anatomical key for Dendrobium species}

Epidermal cell sclerenchymatous:

A. Rectangular to polygonal shaped epidermal cells, c-tetra-monocyclic with circular stomata formosum

B. Square to polygonal shaped epidermal cells, a-tetra-monocyclic with elliptical stomata$-D$. densiflorum

Epidermal cells parenchymatous:

A. Rectangular to polygonal shaped epidermal cells, a, b or c-tetra-monocyclic, eupara-twimonocyclic with elliptical or circular stomata 
Pradhan and Bajracharya: Taxonomic significance of stomatal

- $\quad$ a, b or c-tetra-monocyclic, eupara-twi-monocyclic with elliptical stomata

○ a-tetra-monocyclic -----o. aphyllum, $D$. longicornu

b b-tetra-monocyclic

amoenum, $D$.

- c-tetra-monocyclic $--D$. eriiflorum

○ eupara twi-monocyclic anceps

- a-tetra-monocyclic with circular stomata---------------- -D.heterocarpum, $D$. moniliforme, D. moschatum

B. Square to polygonal shaped epidermal cells, a or b-tetra-monocyclic with elliptical or circular stomata

- $\quad$ a or b-tetra-monocyclic with elliptical stomata

○ a-tetra-monocyclic -----------------------------------D. bicameratum, $D$. denudans

- b-tetra-monocyclic $--D$. crepidatum

- a-tetra-monocyclic with circular stomata ----------D. chrysanthum 


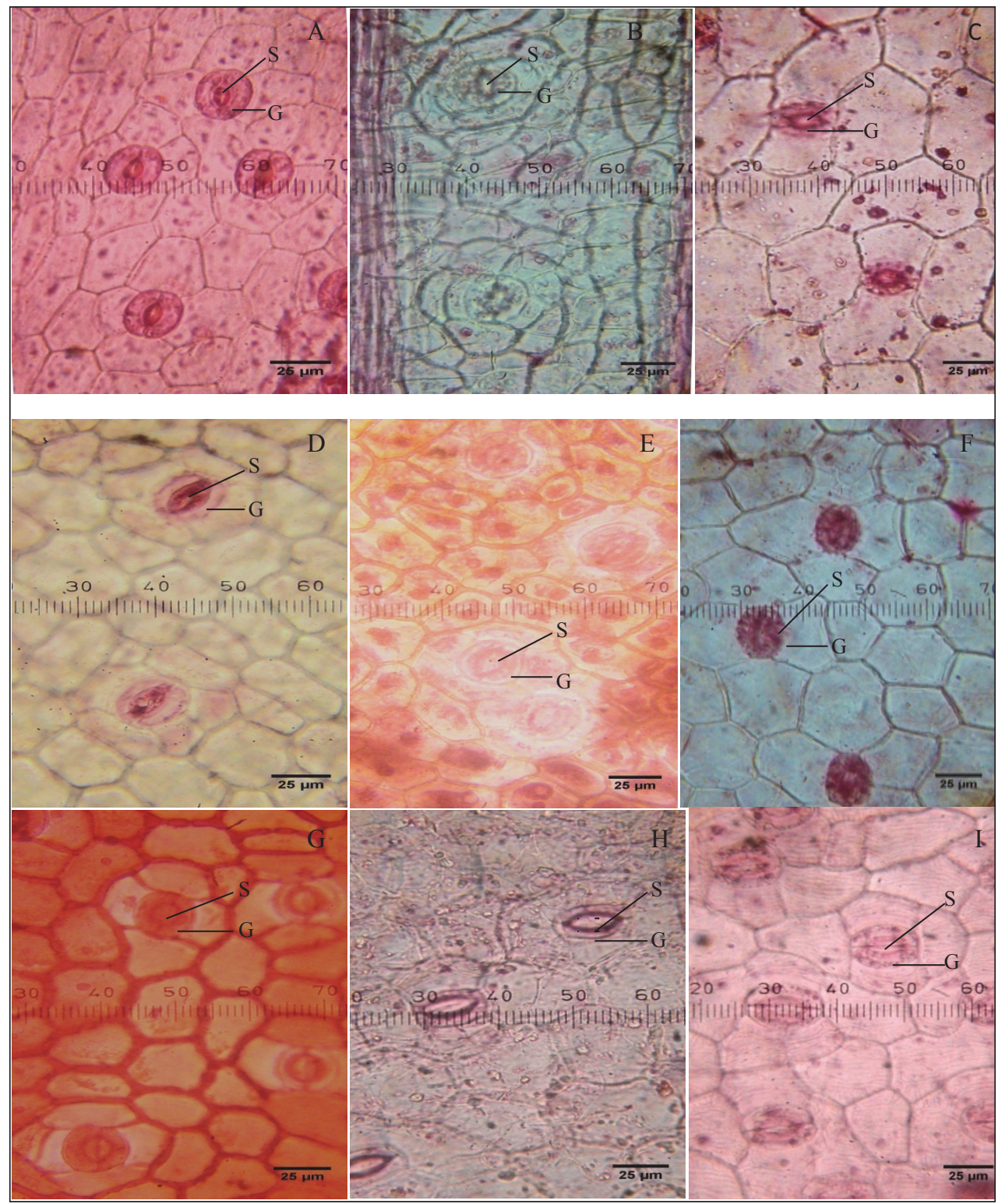

PLATE - I. Stomatal variations in different species of Dendrobium Swartz (Abaxial surface of leaf): A. D. amoenum (b-tetra-monocyclic); B. D. anceps (eupara twi-monocyclic); C. D. aphyllum (a-tetra-monocyclic); D. D. bicameratum (a-tetra-monocyclic), E. D. chrysanthum (a-tetra-monocyclic); F. D. crepidatum (b-tetra-monocyclic); G. D. densiflorum (a-tetra-monocyclic); H. D. denudans (a-tetramonocyclic). I. $D$. erifflorum (c-tetra-monocyclic) (Abbreviations used on photos: $\mathrm{S}=$ Stoma; G = Guard cell). 

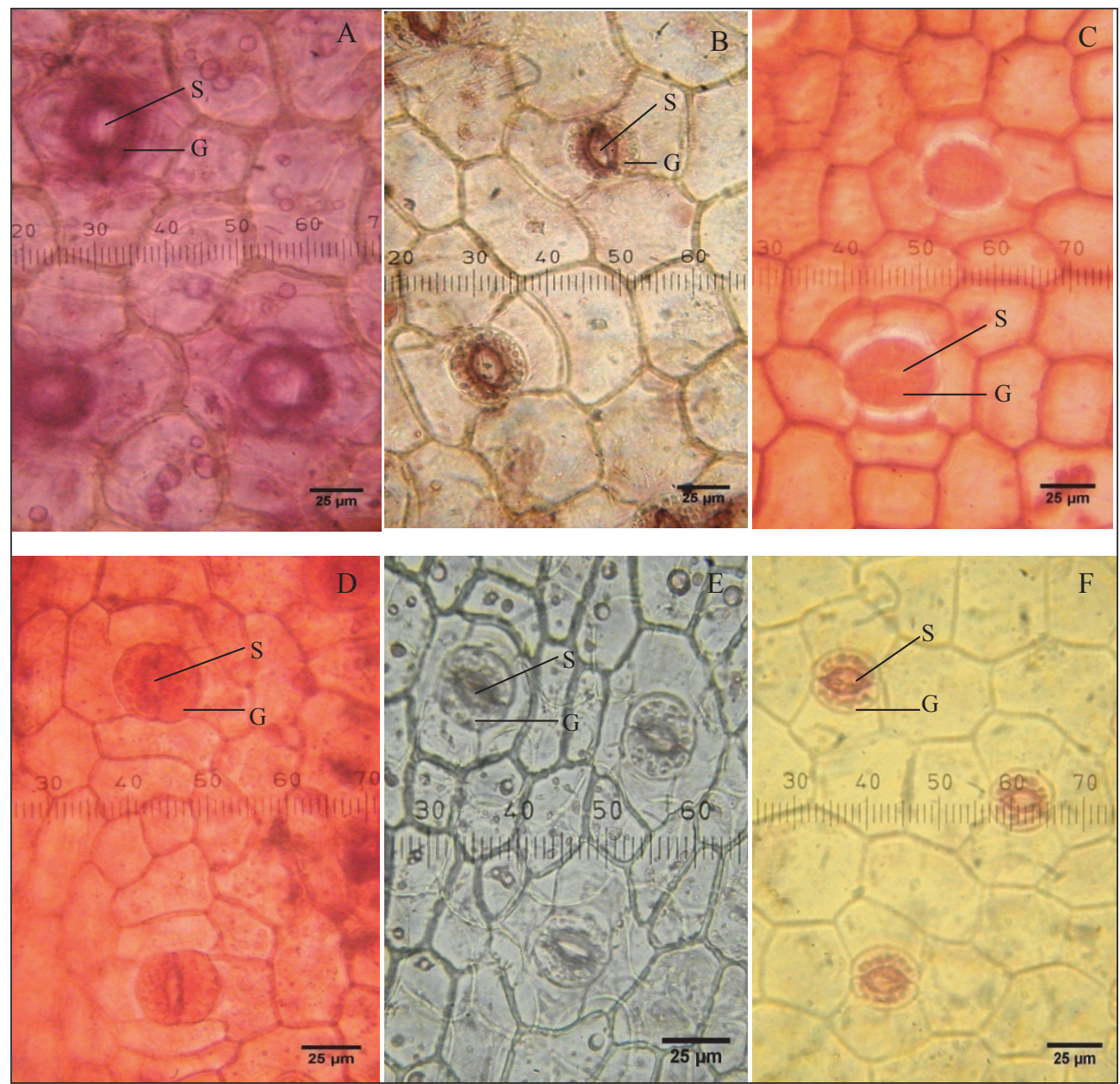

PLATE II. Stomatal variations in different species of Dendrobium Swartz (Abaxial surface of leaf): A. D. formosum (c-tetra-monocyclic); B. D. heterocarpum (a-tetramonocyclic); C. D. Iongicornu (a-tetra-monocyclic); D. D. moniliforme (a-tetramonocyclic); E. D. moschatum (a-tetra-monocyclic); F. D. transparens (b-tetramonocyclic). (Abbreviations used on photos: $\mathbf{S}=$ Stoma; $\mathbf{G}=$ Guard cell).

The present study revealed the stomatal complex of Dendrobium which showed variation in types of epidermal cells, shape, size and types of stomata, stomatal frequency and stomatal index.

All species of Dendrobium showed hypostomatic leaf surface. The leaves are found hypostamic condition in most of orchids (Avadhani et al., 1982). Stomata were found only on the abaxial surface (hypostomatic). This is because these plants are adapted to aerial habitats to minimize 
water loss through stomata (Stebbins \& Khush, 1961). The epidermal cells on the abaxial surface varied from rectangular, squarish, pentagonal, hexagonal to polygonal, irregular with thick or thin walled parenchymatous cells and some species with sclerenchymatous cells. The anticlinal walls of epidermal cell varied from straight-sided to curvilinear.

The shape of stomata varied from elliptical to circular. Elliptical stomata were found in most species of Dendrobium whereas few species had circular stomata. Various modifications of stomatal shape such as elliptical, circular, transversely elliptical and angular are known to exist within Orchidaceae (Rasmussen, 1987). All modifications except the angular shape were found in Dendrobium (Yukawa et al., 1992). Two types of stomatal shape (Stoma I and Stoma II) were reported in Dendrobium (Yukawa et al., 1992). Stoma I is elliptical with a slit-like opening whereas Stoma II is circular with a round to spindle-shaped opening. Similarly two types of stomatal shape i.e. elliptical and circular were found in our study. The size of stomata showed a wide variation from $698.60 \mu \mathrm{m}^{2 \mathrm{t}} \mathrm{O} 1928.48 \mu \mathrm{m}^{2}$. The largest stoma was found in $D$. formosum and the smallest in $D$. amoenum and $D$. longicornu.

Tetracytic type of stomata had been reported in many monocotyledons (Metcalfe, 1961). Mostly three types of stomata paracytic, tetracytic and anomocytric were reported in mostly in monocots (Cheadle, 1953; Stebbins \& Khush, 1961). Stebbins \& Khush (1961) reported that anomocytic type is limited only to the orders closely related to Liliales. Patel (1979) distinguished five types of stomata i.e. tetracytic (a-tetra-monocyclic, b-tetramonocyclic and c-tetra-monocyclic), twicytic, perihaplocytic, anisocytic and hexacytic and each type with four to six subsidiary cells. Rasmussen (1987) distinguished four types of stomata such as tetracytic, anisocytic, cyclocytic and floating stomatal complexes. In the present study tetracytic type (a-tetra-monocyclic, b-tetra-monocyclic and c-tetramonocyclic) was found in most species of Dendrobium whereas eupara twi-monocyclic with hexa-monocyclic types was found in one species ( $D$. anceps). In the stomatal type the monocyclic means the guard cells of the stomata are surrounded by a single cycle of subsdiary cells (Patel, 1979).

The stomatal frequency ranged from $46.15 \mathrm{~mm}^{2}-138.50 \mathrm{~mm}^{2}$. The highest stomatal frequency was found in $D$. moniliforme and lowest in $D$. anceps and $D$. aphyllum. The reduced stomatal frequency was distinctly related with the extent of leaf succulence and more the succulence lesser the frequency of stomata (Goh et al., 1977). Similarly in species from marshy habitat, the highest stomatal frequency was recorded (Ziegenspeck, 1936). The stomatal index ranged from 3.75 to 13.04 . The highest stomatal index was found in $D$. heterocarpum and lowest in $D$. anceps. Similar variation in stomatal index was reported in orchid species (Saadu et al., 2009). The variation in stomatal index could be on account of variation in sunlight intensity (Santosh et al., 2015). A direct correlation between light intensity and stomatal index was observed in orchids (Rasmussen, 1987).

These studies clearly indicated that the micromorphological characters such as distribution of stomata on leaf surface, type of stomata and shape of epidermal cells were found to be taxonomically significant. This parameter will be helpful in solving the existing problems in Dendrobium species. 


\section{AKNOWLEDGEMENTS}

Authors are thankful to National Herbarium and Plant Laboratories, Godawari, Nepal (KATH) and Tribhuvan University Central Herbarium, Kirtipur, Nepal (TUCH) for their cooperation and allowing me to study the herbarium specimens housed over there. My sincere thanks go to Patan Multiple Campus and Amrit Campus for providing the laboratories facilities.

\section{REFERENCES}

ARDITTI, J (1992) Fundamentals of orchid biology. John Wiley and Sons; New York, USA.

AVADHANI, P N; GOH, C J; RAO, A N; ARDITTI, J (1982) Carbon fixation in orchids. In ARDITTI,J (ed) Orchid biology reviews and perspectives. Cornell University Press; Ithaca, New York, USA; Vol. II, pp 173-93.

BAJRACHARYA, D M (2003) The genus Eria Lindley (Orchidaceae) in the Himalayas - a taxonomic revision. Ph. D. Thesis, Central Department of Botany, Tribhuvan University, Kirtipur, Kathmandu, Nepal.

BARANOVA, M (1992) Principles of comparative stomatographic studies of flowering plants. The Botanical Review 58(1): 49-99.

BURNS-BALOGH, P; FUNK, VA(1986) A phylogenetic analysis of Orchidaceae, Smithsonian Contributions to Botany Number 61. Smithsonian Institution Press;Washington D.C., USA.

CARPENTER, K J (2005)Stomatal architecture and evolution in basal angiosperms. Amer J Bot 92 (10): 1595-1615.

CHEADLE, VI (1953) Independent origin of vessels in the monocotyledons \& dicotyledons. Phytomorph 3: 23-44.

CROXDALE, J D (2000) Stomatal patterns in angiosperms. Amer J Bot 87 (8): 1069-1080.

DAVIS, J I (1997) Evolution, evidence and the role of species concepts in phytogenetics. Syst Bot22(2): 373-403.

DRESSLER, R L (1981) The orchids: Natural history and classification. Harvard University Press; Cambridge, MA, USA.

DRESSLER, R L (1993) Phylogeny and classification of the orchid family. Cambridge University Press; MA, USA.

GOH, C J; AVADHANI, P N; LOH, C S; MANEGRAFF, C; ARDITTI, C (1977) Diurnal stomatal and acidic rhythms in orchid leaves. New Phytol 78: 365-72.

HARA, H; STEARN, W T; WILLIAMS, L H J (1978) An enumeration of the flowering plants of Nepal. British Museum of Natural History, London, UK.

KAUSHIK, P (1983) Ecological and anatomical marvels of the Himalayan orchids. Today and Tomorrow's Printers and Publishers; New Delhi, India.

KHASIM, S M; RAO, PR M (1990) Anatomy in relation to taxonomy in some members of Epidendroideae (Orchidaceae). Phytomorphology 40(3-4): 243-250.

KONG, H (2001) Comparative morphology of leaf epidermis in the Chloranthaceae. Bot J Linn Soc 136: 279-294.

METCALFE, C R (1961) The anatomical approach to systematic:General introduction with special reference to recent work on monocotyledons. In BAILEY, D L (ed) Recent advances in botany, University of Toronto Press; Canada; pp 146-150. 
PATEL, J D (1979) New morphological classification of stomatal complexes. Phytomorpholog 29: $218-229$. PEARCE, N R; CRIBB, P J (2002) The orchids of Bhutan. Royal Botanic Garden Edinburgh, Scotland, UK. PRESS, J R; SHRESTHA, K K; SUTTON, D A (2000) Annotated checklist of the flowering plants of Nepal. The Natural History Museum, London, UK.

RASMUSSEN, H (1987) Orchid stomata-structure, differentiation, function and phylogeny. In ARDITTI, $\mathrm{J}$ (ed) Orchid biology-reviews and perspectives Cornell University Press; Ithaca, New York, USA;Vol. 4, pp 105-138.

ROKAYA, M B; RASKOTI, B B; TIMSINA, B; MUNZBERGOVA, Z (2013) An annotated checklist of the orchids of Nepal. Nordic Journal of Botany 31: 511-550.

SAADU, RO; ABDULRAHAMAN, AA; OLADELE, F A (2009) Stomatal complex types and transpiration rates in some tropical tubers species. African Journal of Plant Science 3(5): 107-112.

SALISBURY, E J (1928) On the causes and ecological significance of stomatal frequency with reference to the woodland flora. Phil Trans Ser B 216: 1-65.

SANTOSH, N R; KUMAR, H G P; KRISHNASWAMY, K; SOUZA, G F D (2015) A study on stomatal complex and phenology of certain epiphytic orchids of western ghatts of Karnataka. WJPBT 2 (1): 8-13.

SEIDENFADEN, G (1985). Orchid genera in Thailand XII. Opera Botanica 83: 1-295.

SHAKYA, L R (1999) Revision of the genus Oberonia Lindl. (Orchidaceae) in the Himalayas. Ph. D. Thesis, Central Department of Botany, Tribhuvan University, Kirtipur, Kathmandu, Nepal.

SINGH, V; SINGH, H (1974) Organisation of stomatal complex in some Orchidaceae. Curr Sci43: 490-491.

STACE, C A (1980) Plant taxonomy and biosystematics. Edward Arnold; London, UK.

STEBBINS, G L; KHUSH, G S (1961) Variation in the organization of the stomatal complex in the leaf epidermis of monocotyledons and its bearing on the phylogeny. Amer Jour Bot 48 (1): 51-59.

VIJ, S P; KAUSHAL, P S; KAUR, P (1991) Observations on leaf epidermal features in some Indian orchids: Taxonomic and ecological implications. J Orchid Soc India 5:43-53.

WILKINSON, H P (1979) The plant surface (mainly leaf). In METCALFE, C. R; CHALK, L (eds) Anatomy of the dicotyledons. Clarendon Press; Oxford, UK; Vol. 1, pp 97-165 (2nd edition).

ZIEGENSPECK, H (1936) Orchidaceae. In KIRCHNER, O; LOEW, E; SCHAOTER, C (eds) Lebens geschichte der blutenpflanzen mitteleu ropes. Stuttgart: Eugen Ulmer, Germany (in German). 